\title{
Medición del grado de utilización del enfoque integrado en un curso de inglés para adultos.
}

\section{Ricardo Acosta G.,2, *}

${ }^{1}$ Profesor Titular II, Universidad de Panamá

${ }^{2}$ Decano Honorario, Quality Leadership University

*Autor para correspondencia. E-mail: racosta@qlu.pa

Recibido: 11 de octubre de 2019

Aceptado: 14 de noviembre de 2019

\begin{abstract}
Resumen
Dada la relevancia de la utilización de un enfoque integrado (comunicativo) de enseñanza de las habilidades lingüísticas para el logro de un buen nivel de dominio del idioma en cursos de inglés para adultos, es importante poder determinar, a través de un instrumento confiable, el grado de utilización de dicho enfoque, con el fin de contar con información relevante que posibilite decidir objetivamente las adecuaciones a llevarse a cabo para un mejor desarrollo de las competencias lingüísticas comunicativas propuestas para un determinado curso de inglés. En este artículo presentamos una encuesta original, elaborada por el investigador, que permite evaluar la percepción estudiantil de la utilización en programas de inglés del enfoque integrado (comunicativo). Dicho instrumento fue sometido a pruebas de validez y confiabilidad antes de ser aplicado a un grupo de estudiantes que habían culminado su primer año de estudios en el Centro de Inglés de Quality Leadership University (QLU). La información recabada a través de la encuesta reveló una utilización alta del enfoque integrado en el programa de QLU. La mayoría de los estudiantes encuestados considera que el grado de utilización del enfoque es alto, mientras que una minoría de ellos considera que dicho grado es medio. Además, se logró identificar las áreas de mejora para que la utilización del enfoque integrado para el aprendizaje del inglés sea aún más óptima en los cursos de inglés para adultos de QLU; estas tienen que ver con la necesidad de que se propicie una mayor combinación del aprendizaje del idioma con el aprendizaje de contenidos de otros temas (ciencias, negocios, deportes o historia, por ejemplo), así como con que se establezcan más claramente resultados o metas de aprendizaje relacionados con el uso del idioma para necesidades de la vida diaria, los cuales se espera que los estudiantes cumplan en su clase de inglés.
\end{abstract}

Palabras clave: aprendizaje del inglés, enseñanza de las habilidades lingüísticas, enfoque integrado, enfoque segregado. 


\begin{abstract}
Given the relevance of the use of an integrated (communicative) approach to teaching language skills for the achievement of a good level of language proficiency in adult English courses, it is important to be able to determine, through a reliable instrument, the extent of use of such an approach, in order to have relevant information that would make it possible to objectively decide the adequacies to be carried out for a better development of the communicative language skills proposed for a given English course. In this article, we present an original survey, prepared by the researcher, that allows to evaluate student perception of the use of the integrated (communicative) approach in English programs. This instrument was subjected to validity and reliability tests before being applied to a group of students who had completed their first year of study at the English Center of Quality Leadership University (QLU). The information collected through the survey revealed a high use of the integrated approach in the QLU program. Most of the students that were surveyed consider the degree of use of the approach to be high, while a minority consider such degree to be medium. Also, it was possible to identify areas of improvement, so that the use of the integrated approach to the learning of English may be optimized in the English courses for adults at QLU; these areas have to do with the need to foster a greater combination of language learning with content learning from other topics (sciences, business, sports or history, for example), as well as with more clearly establishing learning goals and/or results related to everyday life, which students are expected to achieve in their English classes.
\end{abstract}

Keywords: English language learning, language skills teaching, integrated approach, segregated approach.

\title{
1 Introducción
}

El grado de uso del enfoque integrado (comunicativo), en vez del segregado (gramatical), de enseñanza de las habilidades lingüísticas (escuchar, hablar, leer y escribir), pudiera estar influyendo sobre los niveles de inglés que logran los estudiantes adultos de nivel principiante que participan en cursos de inglés con el fin de desarrollar un buen nivel de dominio de dicho idioma a través de actividades comunicativas. Es por ello que cobra relevancia el poder determinar, a través de un instrumento confiable, la percepción de los estudiantes del grado de utilización de la enseñanza integrada de las habilidades lingüísticas en programas de inglés para estudiantes de nivel de ingreso principiante. A partir del análisis de la información recabada a través del instrumento, los tomadores de decisiones podrán estar entonces en condiciones de recomendar y/o implementar los correctivos necesarios para que los cursos sean más comunicativos y así poder propiciar el logro de los niveles de aprendizaje de inglés que la sociedad espera. En este artículo compartimos un instrumento original, elaborado por el investigador, que permite evaluar la percepción estudiantil del grado de utilización de la enseñanza integrada en un curso o programa de inglés presencial para adultos.

Para Celce-Murcia (2014), a través de los tiempos, la metodología de la enseñanza de idiomas, en diversos grados de rigurosidad, ha oscilado entre dos tipos de enfoques: conseguir que los estudiantes usen una lengua o conseguir que la analicen. Según Fromkin, Rodman y Hyams (2014), el uso de una lengua involucra entenderla, hablarla, leerla, escribirla y, por consiguiente, comunicarse en ella, mientras que el análisis de una lengua involucra aprender sus reglas gramaticales de morfología, sintaxis, significados (semántica y pragmática), fonética y fonología.

De acuerdo con Harmer (2015), utilizando como referencia el tipo de proceso comunicativo, las habilidades lingüísticas se clasifican en receptivas y productivas; las receptivas son la escucha y la 
lectura (listening and reading) y las productivas son la expresión oral y la escrita (speaking and writing). Basadas en el canal de comunicación utilizado para el proceso comunicativo, dichas habilidades se dividen en habladas y escritas.

Estudios realizados por Celce-Murcia (2014), Jing (2006) y Larsen-Freeman \& Anderson (2016), señalan que en los comienzos de la enseñanza de lenguas, las cuatro habilidades lingüísticas, la escucha, la expresión oral, la lectura y la expresión escrita (listening, speaking, reading, and writing) se enseñaban por separado (segregadamente), además de que los materiales y actividades para la enseñanza eran diseñados usualmente enfocándose en una sola habilidad a la vez, ignorando las otras habilidades. Se creía que el enfoque separado (segregado) en habilidades específicas aceleraba el aprendizaje de un idioma.

Por ello, este enfoque de habilidades segregadas empezó a conocerse como el "enfoque basado en el lenguaje" (Oxford, 2001); en dicho enfoque el lenguaje mismo es el foco central de la enseñanza y no tiene importancia el aprendizaje para la comunicación auténtica (Brown, 2014; Jing, 2006; LarsenFreeman and Anderson, 2016).

En los programas tradicionales de enseñanza del inglés como segunda lengua o como lengua extranjera, las clases son de enfoque segregado de la enseñanza de las destrezas lingüísticas, con cursos de expresión escrita (writing), por ejemplo, divorciados de la expresión oral (speaking) o cursos de escucha (listening) aislados de la lectura (reading). Posiblemente, esto se deba a que los profesores de inglés y los administradores de programas, por cuestiones logísticas, lo consideran más fácil de esta manera, o a que para ellos enfocarse en más de una habilidad lingüística a la vez sea educacionalmente imposible (Oxford, 2001).

Por otro lado, el método Gramática-Traducción (Grammar-Translation Method), el cual dominó la enseñanza de lenguas europeas desde 1840 hasta 1940 y que todavía en forma modificada es ampliamente utilizado en algunas partes del mundo, se enfoca estrictamente en la competencia gramatical y la traducción de un idioma a otro centrándose en la lectura y la escritura, lo cual no enseña a los alumnos el uso del lenguaje para la comunicación en la vida real (Richards \& Rodgers, 2014).

En contraste con el enfoque antes mencionado, en el método Audio-lingual (Audiolingual Method), el cual surgió de la gran atención que recibió la enseñanza de lenguas extranjeras hacia el final de la década de 1950 en los Estados Unidos, entonces se dio gran importancia a las habilidades de expresión oral (speaking) en programas de enseñanza de idiomas basados en los principios de la lingüística estructural y las teorías del aprendizaje conductista. Modelos de enseñanza y aprendizaje de habilidades de escucha (listening skills) adquirieron relevancia para el desarrollo de la expresión oral (LarsenFreeman and Anderson, 2016; Richards and Rodgers, 2014).

Este método concebía a la lengua como auditiva y oral (aural-oral), por lo cual pareció razonable separar las cuatro habilidades (Larsen-Freeman and Anderson, 2016; Tajzad and Namaghi, 2014). Así surgió un modelo de cursos de idiomas y se crearon materiales basados tanto en la separación estructural de las habilidades lingüísticas como en la primacía de la expresión oral (speaking) (Hinkel, 2010). Al igual que sucede con el método Gramática-Traducción, versiones del método Audio-lingual también se siguen utilizando ampliamente en algunas partes del mundo en el presente.

A medida que surgió el enfoque situacional (Situational Approach) en Gran Bretaña, la técnica de enseñanza en el aula llamada PPP (presentación, práctica y producción) se hizo popular. Este modelo 
sostenía que los estudiantes podían utilizar las habilidades lingüísticas por su cuenta en situaciones de la vida real, si se les guiaba a través de la práctica controlada de cada una de las habilidades lingüísticas en la clase (Howatt and Smith, 2014). También se creía que mediante este enfoque se lograría que los estudiantes utilizaran correctamente el idioma estudiado (Richards and Rodgers, 2014). Los investigadores que apoyaban este enfoque sostenían que la enseñanza de cada una de las habilidades lingüísticas por separado permite que los estudiantes obtengan dominio completo sobre una habilidad lingüística como resultado de haberle prestado atención a una habilidad particular a la vez (Jing, 2006).

A pesar de que el término enseñanza situacional de idiomas (Situational Language Teaching) ha caído en desuso, el impacto de este enfoque de enseñanza de idiomas ha sido duradero, ya que dio forma al diseño de muchos renombrados libros de texto y cursos de enseñanza del inglés, particularmente los publicados en el Reino Unido. "Entre los libros más famosos basados en este enfoque se encuentran Streamline English (Hartley and Viney 1978), Access to English (Coles and Lord 1975) y Kernel Lessons Plus (O’Neill 1973). Quizás el mayor legado de este método de enseñanza sea el formato PPP para el planeamiento de clases de idiomas en que muchos docentes de lenguas han sido entrenados al rededor del mundo" (Richards and Rodgers, 2014, p.44).

Pese a todo lo propuesto por estos enfoques y métodos en cuanto a la segregación de la enseñanza de las habilidades lingüísticas, se descubrió que, a pesar de dominar habilidades lingüísticas aisladas, los estudiantes no se podían comunicar en inglés, ya que carecían de competencia comunicativa (communicative competence) (Tajzad \& Namaghi, 2014). Así se llegó a la conclusión de que, aunque fuera posible enseñar una o dos habilidades lingüísticas en ausencia de las otras, el enfoque segregado de enseñanza de las habilidades lingüísticas no prepararía a los estudiantes para la comunicación en la vida diaria, el trabajo o la academia (Celce-Murcia, 2014; Oxford, 2001; Richards \& Rodgers, 2014).

La introducción del concepto de competencia comunicativa (communicative competence) por M. Canale and M. Swain en 1980 causó cambios en las perspectivas de cómo debía enseñarse y usar las habilidades lingüísticas para la comunicación dentro y fuera del aula de clases (Hosseini Breshneh \& Riasati, 2014; Hinkel, 2010). En su libro The Practice of English language Teaching, Harmer (2015) expresa algo similar cuando dice que las habilidades lingüísticas productivas (hablar y escribir) y las receptivas (escuchar y leer) "son dos caras de una misma moneda que no pueden ser separadas a razón del hecho de que una habilidad puede reforzar a otra de varias maneras."

Relacionado a los antecedentes del interés por el desarrollo de la competencia comunicativa (communicative competence), Hinkel (2010) explica que el enfoque comunicativo de enseñanza de lenguas, conocido en inglés como Communicative Language Teaching (CLT) dio lugar a la integración de las cuatro habilidades lingüísticas (escuchar, hablar, leer y escribir) y sus respectivos componentes y que dichas habilidades se complementan. En este caso, las habilidades se integran en la forma en que la gente las utiliza en la comunicación normal (Duff, 2014; Harmer, 2015; Oxford, 2001). Tajzad \& Namaghi (2014) creen que para estudiar un idioma es posible que uno necesite aprender cada habilidad lingüística por separado, pero que las habilidades y componentes del idioma deben ser integrados si a lo que uno aspira es a usar el idioma.

Hinkel (2010) propone que las cuatro habilidades son tanto las metas como los medios para la comunicación. A través de la integración de las habilidades, los estudiantes utilizan el lenguaje auténtico y lo usan para la interacción en la vida real; los alumnos progresan en múltiples habilidades cuando se sigue este enfoque (Hosseini Breshneh \& Riasati, 2014; Oxford, 2001; Valdés Bermúdez, Puig García, Aguirre Cruz, Reyes Martínez, Duarte Martínez y Barata Álvarez, 2015). Además, la 
importancia dada a los significados transmitidos a través del lenguaje, en vez de los aspectos gramaticales y formas de este, incrementa el potencial de los estudiantes de aprender sobre las características funcionales del lenguaje (Larsen-Freeman and Anderson, 2016). Así, aprender a comunicarse tiene prioridad sobre simplemente aprobar un examen académico, y puede ser altamente motivador para estudiantes de todas las edades y orígenes (Huang, 2016; Oxford, 2001).

En la práctica, las destrezas lingüísticas raramente se utilizan aisladamente; por ejemplo, una conversación necesita comprensión y expresión oral. Igualmente, leer, escuchar y tomar notas (escribir) es probable que sea casi tan común como tener una conversación en algunos contextos (Baturay \& Akar, n.d.; Hinkel, 2010; Tajzad \& Namaghi, 2014).

Lo cierto es que la integración de las habilidades es vital para los estudiantes con diferentes estilos de aprendizaje, ya que los extrovertidos pueden practicar el hablar, los introvertidos pueden escuchar o leer, y los alumnos analíticos o visuales pueden ver las oraciones escritas que se construyen (Jing, 2006). Se produce la comunicación óptima en ESL/EFL (inglés como segunda lengua o como lengua extranjera) cuando las habilidades se entrelazan durante la instrucción (Oxford, 2001; Valdés Bermúdez et al., 2015). Tajzad \& Namaghi (2014) sienten que para lograr que los estudiantes se conviertan en usuarios competentes del idioma, las habilidades deben ser integradas desde el primer día.

En los últimos años, las habilidades han sido integradas de manera notable tanto en el método de enseñanza de idiomas conocido como Content Based Language Teaching (CBLT), la enseñanza de idiomas basada en contenidos, como en el método Task Based Language Teaching (TBLT), la enseñanza de idiomas basada en tareas; ambos son considerados como métodos comunicativos (Arslan, 2010).

En el caso de CBLT, los estudiantes aprenden todas las habilidades lingüísticas de una manera integrada a través del aprendizaje de otros temas, por ejemplo, ciencias, matemáticas o estudios sociales. Aquí, el contenido varía en función del nivel de competencia de los alumnos (Brown, 2014; Lightbown \& Spada, 2017; Lightbown, 2014; Stoller, 2002). Lightbown and Spada (2014) explican que las estrategias de aprendizaje de idiomas se integran con el aprendizaje del contenido y el lenguaje. Las habilidades se incorporan con el contenido de acuerdo a tres modelos: el modelo basado en el tema (theme based model), el modelo adjunto (adjunct model) y el modelo protegido (sheltered model). Lightbown \& Spada (2017) añaden que en el modelo basado en el tema "las habilidades lingüísticas se integran con un tema como la delincuencia, la globalización, la educación, entre otros”.

El modelo adjunto coordina cuidadosamente el lenguaje y el contenido, mientras que el modelo protegido simplifica el lenguaje del tema objeto de estudio de acuerdo con el dominio de la lengua de los alumnos. Es importante resaltar que este método se ha desarrollado ampliamente en Europa y es ahora conocido como CLIL (Content and Language Integrated Learning); su nombre deja claro que se trata de la integración del contenido y el lenguaje para el aprendizaje de un idioma (Bentley, 2015).

Un ejemplo más reciente de enfoque mediante el cual se enseñan las habilidades lingüísticas de manera integrada, inspirado en estudios empíricos y que recoge los resultados del trabajo del renombrado lingüista e investigador en temas de adquisición de una segunda lengua Zoltán Dörnyei, es el enfoque comunicativo basado en principios, Principled Communicative Approach (PCA, sus siglas en inglés). En el libro The Principled Communicative Approach: Seven Criteria for Success, publicado por Arnold, Dornyei 
y Pugliese en 2015, se explican y ejemplifican la puesta en práctica en el aula de cada uno de los principios de dicho enfoque.

En las regiones donde se enseña el inglés como idioma extranjero, las oportunidades de comunicación significativa fuera del aula de idiomas son limitadas y existe una gran necesidad de actividades comunicativas que integren las destrezas lingüísticas; esto condujo a la evolución de "Task-based Language Teaching" (TBLT). En TBLT, los estudiantes interactúan y colaboran a través del trabajo grupal o de pareja para completar una tarea específica que asegure el uso del lenguaje de una manera integrada (Hinkel, 2010; Oxford, 2001). Huang (2016) y Larsen-Freeman \& Anderson (2016) coinciden en que TBLT promueve y estimula la integración de habilidades a través de la realización de actividades de la vida diaria que mejoran la competencia comunicativa de los estudiantes porque les ofrece la posibilidad de practicar el idioma objetivo constantemente.

Las actividades pueden ser diversas, desde "conversaciones simuladas en parejas y grupos pequeños, narración de cuentos, descripción de imágenes, reproducción de cualquier incidente" (Akram \& Malik, 2010). Nunan (2010) afirma que "las tareas tienen como objetivo proporcionar ocasiones a los alumnos para experimentar y explorar el lenguaje hablado y escrito a través de tareas de aprendizaje que están diseñadas para involucrar a los estudiantes en el uso auténtico, práctico y funcional del lenguaje" (pág. 41). TBLT permite a los alumnos resolver problemas del mundo real (Calvert and Sheen, 2015). Hasta ahora TBLT es considerada la simulación de aula más cercana a la interacción en la vida real, lo que lo ha convertido en el modelo de enseñanza de idiomas integrado más ampliamente adoptado en la actualidad (Calvert \& Sheen, 2015; Hinkel, 2010; Huang, 2016).

A pesar de que, en el enfoque segregado, cada habilidad lingüística se enseña por separado, la integración de múltiples habilidades se da de manera natural en la clase. De la misma manera como integramos las habilidades cuando utilizamos el idioma en la vida real, resulta irrealista e imposible usar solo una habilidad lingüística en clase únicamente. Por ejemplo, Oxford (2001) explica que en una clase orientada a la lectura (reading), los estudiantes necesitan la habilidad de escucha (listening) para poder entender las instrucciones del profesor y la habilidad de expresión oral (speaking) para discutir la lectura, por lo tanto, integrando estas tres habilidades de manera natural en la clase.

Asimismo, en el caso de los libros de texto, una habilidad en particular podría ser destacada en un libro sobre una habilidad lingüística específica, pero otras habilidades también estarían siendo utilizadas por medio de las actividades presentadas en el libro. Por lo tanto, la implementación del enfoque de habilidades integradas en clases de ESL/EFL puede tener algunas ventajas en contraste con el enfoque de habilidades segregadas. A pesar de que el enfoque de habilidades segregadas puede enseñar a los estudiantes las habilidades lingüísticas de una manera ordenada, la integración de las habilidades les permite usar tales habilidades simultáneamente durante la comunicación. Mientras que en el enfoque segregado la práctica del idioma se da de manera controlada y artificial, en el enfoque integrado, se da la simulación de la comunicación en un contexto de la vida real (Jing, 2006; Tajzad \& Namaghi, 2014).

Además de que las cuatro habilidades (listening, speaking, reading, and writing) se complementan en el aula, el uso simultáneo de estas puede ser muy beneficioso para los estudiantes, ya que posibilita que mejoren uniformemente en las cuatro habilidades. Por ejemplo, una lectura puede proporcionar ideas a los alumnos sobre los que pueden hablar o escribir. Tajzad \& Namaghi (2014) señalan que el enfoque integrado "proporciona a los alumnos tiempo de reflexión" (pág. 97). A los estudiantes se les da la autonomía para reflexionar sobre las ideas del texto de lectura o de escucha y relacionar las mismas 
con sus propios conocimientos, opiniones o experiencias a través del habla o la escritura. Se ha descubierto que la lectura es buena para el desarrollo de vocabulario útil y que los estudiantes tienen un mejor desempeño en la escritura (writing) cuando otras habilidades y sub-habilidades lingüísticas han sido integradas también (Akram \& Malik, 2010; Cushing, 2014; Lyutaya, 2011).

Con el enfoque tradicional segregado los estudiantes tienden a estar menos motivados, ya que se les enseña el idioma como un tema en sí. A través de la integración de las habilidades se trata el idioma como un medio para la interacción, lo cual impulsa la motivación y la confianza de los estudiantes, quienes en consecuencia pueden expresar sus propias ideas y puntos de vista sin temor al docente (Duff, 2014; Huang, 2016). Tajzad \& Namaghi (2014) explican que la integración de las habilidades reduce el nivel de estrés y ansiedad de los estudiantes al involucrarlos en la comunicación real de ideas en lugar de prácticas mecanizadas de habilidades lingüísticas y sus componentes.

De hecho, el enfoque segregado se centra en la forma, el dominio de la lengua, la exactitud (accuracy). Sin embargo, el enfoque integrado se centra en el significado, la utilización del lenguaje para la comunicación, la fluidez. El primer enfoque es estresante en la medida en que estimula la memorización y el dominio de las formas de la lengua, mientras que el segundo es menos estresante y cognitivamente menos exigente, ya que se centra en expresar e intercambiar ideas (Akram \& Malik, 2010; Tajzad \& Namaghi, 2014).

El enfoque de habilidades segregadas se centra en el docente, que es quien establece las tareas y actividades. Sin embargo, el enfoque integrado de habilidades lingüísticas da a los estudiantes autonomía y se centra en los estudiantes, ya que son libres de construir y negociar significados durante la lectura y la escucha, así como intercambiar y compartir ideas durante la escritura y el habla. Esto desafía y reconstruye el papel del profesor en la clase, además de que involucra un aumento del tiempo que habla el estudiante y una disminución del tiempo que habla el profesor. Tajzad \& Namaghi (2014) observan que la integración de las habilidades conduce a "la re-conceptualización del papel del docente" (p. 96). Por otro lado, Banegas (2012), Huang (2016) y Jing (2006) coinciden en que la integración exitosa de las habilidades puede hacer que las clases sean dinámicas al involucrar a los estudiantes en diversas actividades que les dan amplias oportunidades para la interacción y la comunicación y que les motivan a aprender.

Las habilidades lingüísticas pueden ser integradas en diferentes maneras, basadas en los estudiantes y en el contexto de la clase. De acuerdo a Harmer (2015) y Hinkel (2010), la incorporación de dos habilidades en el mismo medio lingüístico es el enfoque de enseñanza integrado más simple y básico; es decir, en el medio oral que incluye escuchar y hablar, o en el medio escrito que incluye la lectura y la escritura. Esta integración puede darse muy naturalmente en clase sin mucho esfuerzo del profesor y de los estudiantes. Además, las habilidades receptivas de escuchar y leer (listening and reading) no pueden funcionar solas en el aula ESL/EFL.

Por lo tanto, una habilidad productiva, como hablar (speaking) o escribir (writing), puede ser incorporada con una habilidad receptiva, como escuchar (listening) o leer (reading). Por ejemplo, en el caso de habilidades de escucha (listening), los principiantes pueden escribir o hablar con el fin de expresar lo que han entendido del texto escuchado. La integración más compleja ocurre cuando más de dos habilidades son integradas; la integración puede ser de entre tres a cuatro habilidades.

Según Hinkel (2010), la integración compleja ocurre cuando la clase es una combinación de habilidades lingüísticas múltiples. Por ejemplo, el uso de material de escucha o de lectura con el fin de promover 
una actividad de hablar y otra de escribir. En este caso, el contenido y el tema de las tareas o actividades influirán en las habilidades que se integrarán (Amin Mekheimer \& Shabieb Aldosari, 2013). Hinkel (2010) también está de acuerdo en que en situaciones complejas de integración de las destrezas lingüísticas en el aula los materiales se organicen basándose en el mismo tema. Por lo tanto, se trata de un enfoque más 'basado en el discurso' donde las habilidades se organizan de manera que se facilite la cohesión temática, en lugar de centrarse en elementos de una habilidad segregada específica sin ningún contexto (Celce-Murcia \& Olshtain, 2014).

Existe la necesidad de más investigación sobre lo que los docentes deben hacer para integrar las habilidades con éxito en la clase. De los estudios disponibles, se puede discurrir que los profesores necesitan familiarizarse con las formas de integrar las habilidades lingüísticas en el aula de ESL/EFL, tomando en consideración que principalmente las habilidades se han integrado bajo métodos como CBLT y TBLT, o combinando ambos (Richards \& Rodgers, 2014). Igualmente, los docentes pueden examinar objetivamente su enfoque actual hacia la enseñanza de las habilidades lingüísticas y evaluarlo. Es responsabilidad de los profesores reflexionar sobre las posibles formas de integrar más habilidades para hacer sus clases más dinámicas, así como centrarse en las necesidades de los estudiantes y enseñarles el uso auténtico de la lengua (Brown, H.D., 2014; Murphy, 2014).

En el presente artículo presentamos un resumen de la experiencia de la validación y aplicación de la encuesta original elaborada por el investigador con el fin de medir la percepción estudiantil del grado de utilización del enfoque integrado de enseñanza de las habilidades lingüísticas en un programa o curso presencial de inglés para adultos.

\section{Materiales y Métodos}

Una encuesta de opción múltiple (ver Anexo 1), originalmente elaborada, se llevó a cabo entre 21 participantes de un curso de inglés para adultos ofrecido en Quality Leadership University (QLU), donde acuden principalmente estudiantes provenientes de comunidades de Panamá Centro (y en menor proporción de San Miguelito, Panamá Este y Panamá Oeste) en edades entre 18 y 55 años de ambos géneros, quienes son principalmente de clase media (baja y alta) y estudian en el turno nocturno. No se trabajó con una muestra sino con el universo de los estudiantes (21) de uno de los grupos que habían culminado su primer año de participación en el programa de QLU, en el cual las habilidades lingüísticas (escuchar, hablar, leer y escribir) se enseñan de manera integrada (comunicativa).

El grupo de estudio estuvo conformado por 7 participantes del género masculino y 14 del género femenino, en su mayoría provenientes de Panamá Centro (12), seguidos de los provenientes de Panamá Este (cuatro), San Miguelito (tres), Panamá Oeste (uno) y Panamá Norte (uno). Son relativamente jóvenes, con edades en un rango de 18 a 22 años (cuatro), de 23 a 27 años (dos), de 28 a 32 años (tres), de 33 a 37 años (dos), de 38-42 años (cuatro), y de 43 a 47 años (uno); cuatro de los participantes se encuentran en un rango de edad de 48 a 52 años, mientras que solo uno tiene 53 o más años.

Para lograr el propósito de conocer el grado de utilización del enfoque integrado (comunicativo) de enseñanza de las habilidades lingüísticas en el programa objeto del estudio, no se hicieron observaciones ni entrevistas exhaustivas, sino que se utilizó una encuesta que nos permitió recabar 
información sobre la percepción de los participantes del estudio en cuanto a la utilización del mencionado enfoque en el curso de inglés para adultos de QLU.

En la encuesta se buscó determinar la percepción de los estudiantes del grado de utilización del enfoque integrado (bajo, medio o alto) en lo concerniente a la presencia de las características principales del enfoque (items 1-11), el rol que desempeña el profesor (items 12-14), el rol que desempeña el estudiante (ítems 15-17) y las actividades propias del enfoque integrado (ítems18-20) en el programa de QLU.

La encuesta está constituida por un total de 20 ítems, los cuales pueden ser respondidos de acuerdo con las siguientes opciones, a las cuales se les asignó un valor de 1 a 5 : muy de acuerdo $=5$, de acuerdo $=4$, parcialmente de acuerdo $=3$, en desacuerdo $=2$ y totalmente en desacuerdo $=1$. Los puntajes totales representativos para cada grado de utilización del enfoque son los siguientes: de 81 a 100: alto; de 61 a 80: medio; de 60 o menos: bajo.

Para determinar la validez de la encuesta, se elaboró un cuestionario con preguntas (ver Anexo 2) que fueron respondidas por un panel de 17 expertos de la enseñanza del inglés y que, además de responder con SÍ o NO, también podían expresar sus comentarios por escrito sobre cómo los instrumentos podrían ser mejorados. Las respuestas, ya afirmativas ya negativas, de cada experto fueron llevadas a una matriz en donde cada respuesta afirmativa de un juez tenía un valor de 1 y cada negativa un valor de 0 . Una vez sumados los números totales de acuerdos y desacuerdos de los jueces, se procedió a llevar a cabo una prueba de concordancia entre los jueces, utilizando la siguiente fórmula: $\mathbf{b}=(\mathbf{T a} \div$ $\mathbf{T a}+\mathbf{T d}) \times$ 100. Donde Ta representa el número total de acuerdos entre los jueces; Td, el número total de desacuerdos y b, el grado de concordancia significativa. El porcentaje que arrojó la aplicación de la fórmula fue de $94.957 \%$ de acuerdo entre los jueces, lo cual es excelente. Los detalles de los procedimientos antes mencionados forman parte del Anexo 2.

En cuanto a la confiabilidad de la encuesta, se llevó a cabo una prueba piloto de la misma, en la que participaron 41 individuos. Los resultados obtenidos se sometieron a una prueba de Alfa de Cronbach, la cual arrojó que la encuesta tenía una fiabilidad excelente de ,974. La prueba se realizó a través del software estadístico SPSS.

En cuanto a los métodos utilizados para el análisis de los datos, para los resultados de la encuesta se calcularon las medidas de tendencia central y se presentaron los resultados en una tabla para cada factor, con el fin de descubrir las principales propiedades y características clave de los resultados obtenidos a través de la encuesta.

\section{Resultados, análisis y/o discusión}

Un grupo de 21 estudiantes de Quality Leadership University (QLU), quienes participaron del programa de inglés para adultos de QLU durante un año, tomaron la encuesta original disponible en el Anexo 1, con la cual se midió la percepción de estos estudiantes sobre el grado de utilización del enfoque integrado de enseñanza de las habilidades lingüísticas en el curso de inglés para adultos de QLU. A continuación, los resultados obtenidos. 
Tabla 1.1 Grado de utilización del enfoque integrado en el Programa de QLU

\begin{tabular}{|l|l|l|}
\hline & Enfoque del programa & Total \\
& INTEGRADO & \\
\hline $\begin{array}{l}\text { Grado de utilización del ALTO, 81-100 puntos } \\
\text { enfoque integrado }\end{array}$ & 19 & 19 \\
Total & 2 & 2 \\
\hline
\end{tabular}

Fuente: Autoría del investigador.

Gráfica 1. Grado de utilización del enfoque integrado en el Programa de QLU

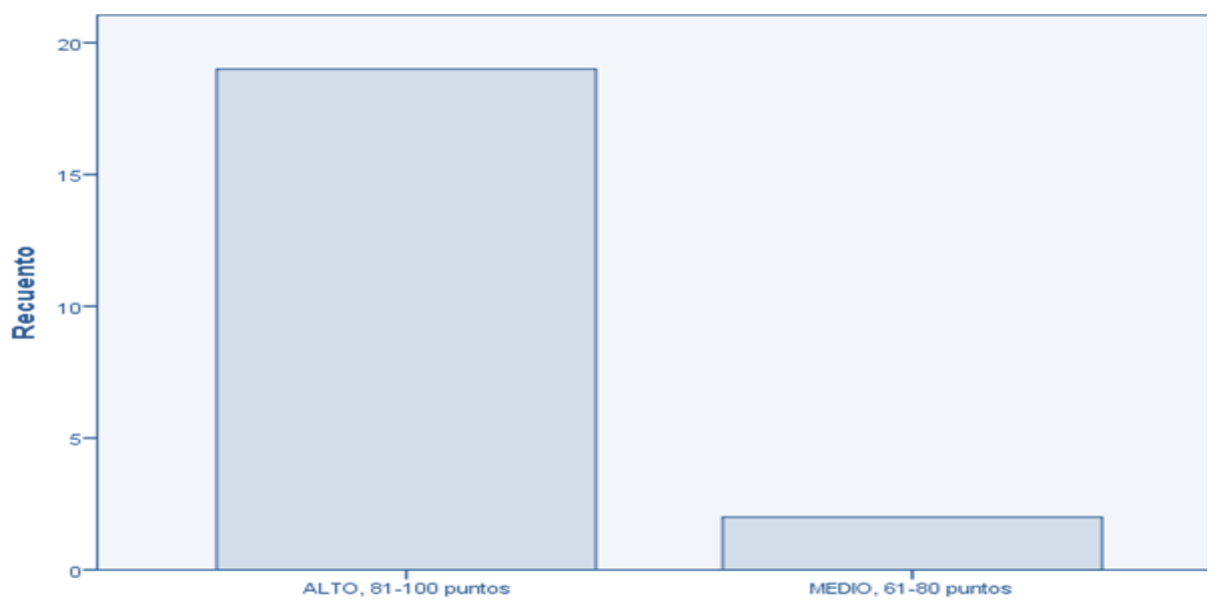

Fuente: Autoria del investigador.

La mayoría de los estudiantes, 19 de ellos, considera que el grado de utilización del enfoque integrado en el programa de inglés de QLU es alto, mientras que una minoría, solo 2 de ellos, considera que el grado de utilización del enfoque es medio, lo cual también denota una percepción favorable del grado de utilización del mencionado enfoque.

A continuación, presentamos las tablas en donde se ilustra la manera como los estudiantes respondieron las preguntas de la encuesta. Los ítems del 1-11 de la Tabla 2, miden la presencia en el programa respectivo de las características principales del enfoque integrado; los del 12-14 de la Tabla 3 miden el rol del profesor; los del 15-17 de la Tabla 4 miden el rol de los estudiantes; y los del 18-20 de la Tabla 5 miden el uso de las actividades propias del enfoque integrado.

Como se observa en la Tabla 2, la percepción de los estudiantes es altamente positiva respecto a la presencia en el programa de inglés cursado de las características principales del enfoque integrado, con la excepción de lo planteado en la pregunta 8 , donde en promedio los estudiantes perciben la presencia de ese factor particular como media. La media de 3.7143 para las respuestas de los estudiantes a dicha pregunta indica un grado de acuerdo medio sobre si en el programa de QLU se combina el aprendizaje 
del idioma con el aprendizaje de contenidos de otras asignaturas (ciencia, geografía, o historia, por ejemplo) en la clase de inglés. Las respuestas a la pregunta 8 dejan claro que la enseñanza del inglés basada en contenidos es menos prevalente que la instrucción basada en tareas (TBLT), pero que la misma es utilizada también en el programa de QLU.

Tabla 2. Presencia de las características principales del enfoque integrado en el Programa de QLU.

\begin{tabular}{|c|c|c|c|c|c|}
\hline & $\mathrm{N}$ & Mínimo & Máximo & Media & Desv. típ. \\
\hline $\begin{array}{l}\text { 1.¿Su clase de inglés se enfoca en las } \\
\text { funciones de dicho idioma y los } \\
\text { significados que se pueden transmitir } \\
\text { a través del mismo? } \\
\text { 2.¿En su clase se enfatiza la } \\
\text { interacción en inglés? } \\
\text { 3.¿Durante las clases, se utilizan } \\
\text { materiales e información auténtica en } \\
\text { inglés (periódicos, panfletos, mapas, } \\
\text { películas, revistas, } \\
\text { noticias, etc.)? } \\
\text { 4.¿Se aprende inglés haciendo y a } \\
\text { través de la práctica directa en su } \\
\text { clase? } \\
\text { 5. ¿El aprendizaje centrado en el } \\
\text { estudiante se practica en su clase de } \\
\text { inglés? En otras palabras, ¿son los } \\
\text { estudiantes mayormente los que } \\
\text { hablan y llevan a cabo diferentes } \\
\text { actividades en vez de tener al docente } \\
\text { hablando o explicando algo todo el } \\
\text { tiempo? } \\
\text { 6.¿En su clase se hacen esfuerzos por } \\
\text { practicar la comunicación auténtica } \\
\text { (para el mundo real) en inglés? } \\
\text { 7.¿El trabajo en parejas y el trabajo en } \\
\text { grupos son comunes en su clase de } \\
\text { inglés? } \\
\text { 8.¿Se combina el aprendizaje del } \\
\text { idioma con el aprendizaje de } \\
\text { contenidos de otros temas (ciencias, } \\
\text { negocios, deportes o historia, por } \\
\text { ejemplo) en su clase de inglés? } \\
\text { 9. ¿Su clase de inglés se enfoca en el } \\
\text { intercambio de información a través } \\
\text { de la comunicación? }\end{array}$ & 21 & $\begin{array}{l}3.00 \\
4.00\end{array}$ & $\begin{array}{l}5.00 \\
5.00\end{array}$ & $\begin{array}{l}3.7143 \\
4.4286\end{array}$ & $\begin{array}{l}.59761 \\
.58959\end{array}$ \\
\hline
\end{tabular}




\begin{tabular}{|c|c|c|c|c|c|}
\hline $\begin{array}{l}\text { 10. ¿Existen resultados o metas de } \\
\text { aprendizaje relacionados con el uso } \\
\text { del idioma para necesidades de la vida } \\
\text { diaria que han sido establecidos y que } \\
\text { se espera que cumpla en su clase de } \\
\text { inglés? } \\
\text { 11.¿En su clase se practican y se } \\
\text { integran las habilidades de entender, } \\
\text { hablar, leer y escribir en inglés a } \\
\text { través del aprendizaje cooperativo } \\
\text { entre estudiantes? } \\
\mathrm{N} \text { válido (según lista) }\end{array}$ & 21 & 3.00 & 5.00 & 4.0000 & .77460 \\
\hline
\end{tabular}

Fuente: Autoria del investigador.

En la Tabla 3, podemos observar que la percepción de los estudiantes de QLU es favorable en cada uno de los tres temas relacionados con el rol del profesor en la enseñanza de las habilidades lingüísticas de manera integrada en el programa cursado. Las medias de las respuestas (entre 4.333 y 4.619) son altas y denotan que los estudiantes consideran al profesor/a un participante y facilitador/a importante en el proceso de enseñanza y aprendizaje.

Tabla 3.2 Rol que desempeña el profesor en el Programa de QLU

\begin{tabular}{|l|l|l|l|l|l|}
\hline & $\mathrm{N}$ & Mínimo & Máximo & Media & Desv. típ. \\
\hline $\begin{array}{l}\text { 12. ¿El/La profesor/a es un } \\
\text { facilitador/a de la comunicación en su } \\
\text { clase de inglés? }\end{array}$ & 21 & 4.00 & 5.00 & 4.5238 & .51177 \\
$\begin{array}{l}\text { 13. ¿El/La profesor/a fomenta el uso } \\
\text { del inglés y es un/a participante activo/a } \\
\text { en la clase? } \\
\begin{array}{l}\text { 14. ¿El/La profesor/a proporciona } \\
\text { retroalimentación continua con respecto } \\
\text { a los objetivos de aprendizaje de la clase? } \\
\text { N válido (según lista) }\end{array}\end{array}$ & 21 & 3.00 & 5.00 & 4.6190 & .58959 \\
\hline
\end{tabular}

Fuente: Autoría del investigador.

En la Tabla 4, podemos observar que la percepción de los estudiantes de los cursos de inglés para adultos de QLU sobre su rol en el programa de inglés cursado revela un grado alto de utilización del enfoque integrado; sus respuestas entre las medias de 3.952 y 4.333 indican un grado alto de utilización tanto del aprendizaje colaborativo como del autónomo (aunque en menor proporción) en el programa cursado. En el caso de la pregunta 17, los estudiantes perciben que, aunque esté presente también, el aprendizaje autónomo no es tan prevalente en el programa de QLU como las actividades comunicativas y el aprendizaje cooperativo entre estudiantes. 
Tabla 4. Rol que desempeña el estudiante en el Programa de QLU

\begin{tabular}{|c|c|c|c|c|c|}
\hline & $\mathrm{N}$ & Mínimo & Máximo & Media & Desv. típ. \\
\hline $\begin{array}{l}\text { 15.¿En su clase de inglés los } \\
\text { estudiantes son participantes } \\
\text { comunicativos activos? } \\
\text { 16.¿Los estudiantes colaboran } \\
\text { entre sí para el aprendizaje del } \\
\text { idioma en su clase de inglés? } \\
\text { 17. ¿Además del aprendizaje en } \\
\text { colaboración, los estudiantes de } \\
\text { su clase de inglés practican el } \\
\text { aprendizaje autónomo (iniciado y } \\
\text { dirigido por ellos mismos)? } \\
\mathrm{N} \text { válido (según lista) }\end{array}$ & 21 & $\begin{array}{l}3.00 \\
3.00\end{array}$ & $\begin{array}{l}5.00 \\
5.00\end{array}$ & $\begin{array}{l}4.0952 \\
4.3333\end{array}$ & $\begin{array}{l}.43644 \\
.57735 \\
.66904\end{array}$ \\
\hline
\end{tabular}

Fuente: Autoría del investigador.

Como se observa en la Tabla 5 a continuación, la percepción de los estudiantes sobre el uso de las actividades propias del enfoque integrado en el programa de inglés de QLU, evidenciada por sus respuestas con medias de 4.095 a 4.285, también denota un grado alto de utilización del mencionado enfoque.

Tabla 5. Actividades propias del enfoque integrado en el Programa de QLU

\begin{tabular}{|l|l|l|l|l|l|}
\hline & $\mathrm{N}$ & Mínimo & Máximo & Media & Desv. típ. \\
\hline $\begin{array}{l}\text { 18. ¿Se practican en su clase de inglés } \\
\text { actividades de aprendizaje } \\
\text { colaborativo en que se debe buscar, } \\
\text { intercambiar }\end{array}$ & 21 & 3.00 & 5.00 & 4.0952 & .53896 \\
información? y compartir \\
$\begin{array}{l}\text { 19. ¿Se dan en su clase de inglés } \\
\text { actividades orientadas al desempeño } \\
\text { de tareas o actividades de la vida real? } \\
\begin{array}{l}\text { 20. ¿En su clase de inglés se llevan a } \\
\text { cabo actividades dirigidas a la práctica } \\
\text { del inglés y al desarrollo de fluidez en } \\
\text { dicha lengua? } \\
\text { N válido (según lista) }\end{array}\end{array}$ & 21 & 3.00 & 5.00 & 4.1429 & .47809 \\
\hline
\end{tabular}

Fuente: Autoría del investigador. 


\section{Conclusiones}

Esta investigación abordó la utilización de un instrumento para medir la percepción estudiantil del grado de utilización del enfoque integrado de enseñanza de las habilidades lingüísticas en un curso de inglés para adultos, con el fin de determinar lo que se debe mejorar para optimizar el aprendizaje del idioma por parte de los participantes en programas en que las habilidades lingüísticas se enseñan de manera integrada (comunicativa). A través de la encuesta original elaborada por el investigador (Anexo 1), recogimos las percepciones (luego de un año de participación en el programa de inglés) de los participantes del estudio con respecto al grado de utilización del enfoque integrado en el programa de inglés para adultos de enfoque comunicativo de Quality Leadership University (QLU).

Por medio de los resultados de la encuesta se logró constatar la utilización extensiva de un enfoque comunicativo para el aprendizaje y la enseñanza del inglés en el programa de QLU. La mayoría de los estudiantes que completaron su año de estudio en dicho programa considera que el grado de utilización del enfoque integrado (comunicativo) de enseñanza de las habilidades lingüísticas es alto, mientras que una minoría de ellos considera que el grado de utilización del mencionado enfoque es medio, lo cual también es favorable. Por otro lado, se logró la identificación de áreas de mejora para que el aprendizaje del inglés sea aún más óptimo, que en el caso de los participantes de los cursos de inglés de QLU tienen que ver con con la necesidad de que se propicie una mayor combinación del aprendizaje del idioma con el aprendizaje de contenidos de otros temas (ciencias, negocios, deportes o historia, por ejemplo), así como con que se establezcan más claramente los resultados o metas de aprendizaje relacionados con el uso del idioma para necesidades de la vida diaria que se espera que los estudiantes cumplan en su clase de inglés.

Para la implementación de la enseñanza integrada de las habilidades lingüísticas es necesario no solo que la presencia de las características principales del enfoque integrado sea entre media y alta, sino también que tanto los estudiantes como el/la profesor(a) jueguen el rol correcto para que las actividades propias del enfoque integrado (comunicativo) se den de manera regular y fluida. Es por ello que, con el fin de conocer el grado de utilización del enfoque integrado, estos aspectos deben ser medidos y luego analizados para así poder realizar oportunamente cualquier adecuación necesaria para el éxito de los estudiantes en la mejora de su nivel de dominio del inglés. Reiteramos que el grado de utilización del enfoque integrado (comunicativo) idealmente se debe mantener entre un nivel medio y alto, tomando en cuenta que la implementación de dicho enfoque está principalmente en manos de las instituciones y de los profesores. Los estudiantes solo podrán asumir el rol que les corresponde en este tipo de enfoque en la medida en que los profesores y las instituciones donde toman cursos de inglés propicien el aprendizaje de las habilidades lingüísticas de manera integrada y el uso del lenguaje para la vida real.

\section{Referencias}

Akram, A. and Malik, A. (2010). Integration of Language Learning Skills in Second Language Acquisition. International Journal of Arts and Sciences, 3(14), 231-240. Disponible en http:// citeseerx.ist.psu.edu/ viewdoc/download? doi $=10.1 \cdot 1.301 .4890 \&$ rep $=$ rep1\&type $=$ pdf

Amin Mekheimer, M. and Shabieb Aldosari, H. (2013). Evaluating an Integrated EFL 
Teaching Methodology in Saudi Universities: A Longitudinal Study. Journal of Language

Teaching and Research, Vol. 4, No. 6, pp. 1264-1276, November 2013.

Arnold, J., Dornyei, Z. \& Pugliese, C. (2015). The Principled Communicative

Approach: Seven Criteria for Success. London: Helbling Languages

Arslan, A. (2010). Implementing a Holistic Teaching in Modern ELT Classes: Using

Technology and Integrating Four Skills. International Journal of Human

Sciences.

Banegas, D. (2012). Integrating content and language in English language teaching in secondary education: models, benefits and challenges, Studies in Second Language Learning and Teaching 2/1: 111-136: Disponible en: https://repozytorium.amu.edu.pl/jspui/bitstream/10593/2907/1/SSLLT\%202(1)\%20111136\%20Banegas.pdf

Baturay, M. H. \& Akar, N. (s/f). A New Perspective for the Integration of Skills to Reading. 16- 27. Disponible en http://dergiler.ankara.edu.tr/dergiler/27/761/ 9653.pdf

Bentley, Kay. (2015). The TKT Course. CLIL Model. Cambridge University Press.

Brown, H. D. (2014). Principles of Language Learning and Teaching (6 $6^{\text {th }}$ Edition). Pearson.

Celce-Murcia, M. (2014). An Overview of Language Teaching Methods and Approaches. En Celce-Murcia, M., Brinton D., and M. Snow. Teaching English as a Second or Foreign Language (4 ${ }^{\text {th }}$ Edition). Boston, MA: Heinle Cengage Learning.

Celce-Murcia, M and Olshtain, E. (2014). Teaching Language through Discourse. En Celce-Murcia, M., Brinton D., and M. Snow. Teaching English as a Second or Foreign Language $\left(4^{\text {th }}\right.$ Edition). Boston, MA: Heinle Cengage Learning.

Celce-Murcia, M., Brinton D., and M. Snow. (2014). Teaching English as a Second or Foreign Language ( $4^{\text {th }}$ Edition). Boston, MA: Heinle Cengage Learning.

Cushing, S. (2014). Considerations for Teaching Second Language Writing. En Celce-

Murcia, M., D. Brinton, and M. Snow. Teaching English as a Second or Foreign Language $\left(4^{\text {th }}\right.$

Edition). Boston, MA: Heinle Cengage Learning.

Duff, P.A. (2014). Communicative Language Teaching. En Celce-Murcia, M., D.

Brinton, and M. Snow. Teaching English as a Second or Foreign Language (4 ${ }^{\text {th }}$ Edition). Boston,

MA: Heinle Cengage Learning.

Fromkin, V., R. Rodman, and N. Hyams. (2014). An Introduction to Language $\left(10^{\text {th }}\right.$

Edition). Boston, MA: Wadsworth Cengage Learning.

Harmer, J. (2015). The Practice of English Language Teaching. Pearson.

Hinkel, E. (2010). Integrating the Four Skills: Current and Historical Perspectives. En

The Oxford Handbook of Applied Linguistics (2 ed.). Oxford University Press.

Hosseini Breshneh, A. y Riasati, M.J. (2014). Communicative language teaching:

Characteristics and principles. International Journal of Language and Applied Linguistics World, 6 (4), 436-445.

Howatt, A. P. R. and Smith, R. (2014). The History of Teaching English as a Foreign

Language, from a British and European Perspective. Language \& History Vol. 57, I.

Huang, D. (2016). A Study on the Application of Task-based Language Teaching 
Method in a Comprehensive English Class in China. Journal of Language Teaching and Research, Vol. 7.

Jing, W. (2006). Integrating Skills for Teaching EFL-Activity Design for the

Communicative Classroom. Sino-US English Teaching, Dec. Volume 3, No.12.

Larsen-Freeman, D. y Anderson, M. (2016). Techniques and principles in language teaching. 3a Edición. New York: Oxford University Press.

Lightbown, P., and Spada, N. (2017). How Languages Are Learned. Oxford University Press.

Lyutaya, Tatiana. (2011). Reading Logs: Integrating Extensive Reading with Writing Tasks. English Teaching Forum, N¹.

Mathew, N. G., \& Alidmat, A. O. H. (2013). A study on the Usefulness of AudioVisual Aids in EFL Classrooms: Implications for Effective Instruction. International Journal of Higher Education, 2(2). http://dx.doi.org/10.5430/ijhe.v2n2p86

Murphy, J. (2014). Reflective Teaching: Principles and Practices. En Celce-Murcia, M., D. Brinton, and M. Snow. Teaching English as a Second or Foreign Language (4 $4^{\text {th }}$ Edition). Boston, MA: Heinle Cengage Learning.

Nunan, D. (2010). Task Based Language Teaching. Online Publication. Oxford University Press.

Oxford, R. (2001). 'Integrated Skills in the ESL/EFL Classroom'. ERIC Digest. ED456670.

Richards, J.C. \& Rodgers, T.S. (2014). Approaches and methods in language teaching. Cambridge: Cambridge University Press.

Tajzad, M. \& Namaghi, S. A. O. (2014). Exploring EFL Learners' Perceptions of Integrated Skills Approach: A Grounded Theory. English Language Teaching, 7(11), 92-98. Recuperado de http:/ files.eric.ed.gov/fulltext/EJ 1075939.pdf.

Valdés Bermúdez, R. V., A. Puig García, A. Aguirre Cruz, E. Reyes Martínez, A. Duarte Martínez y A. Barata Álvarez (2015). Manual didáctico sobre la integración de las cuatro habilidades lingüísticas en la enseñanza del inglés.

EDUMECENTRO vol.7 no.4 Santa Clara oct.-dic. 2015 
ANEXO 1: Encuesta sobre grado de utilización del enfoque integrado de enseñanza de las habilidades lingüísticas en un curso de inglés (Nota: Encuesta original elaborada por el investigador, Dr. Ricardo Acosta G. No podrá ser reproducida sin el permiso y debido reconocimiento de su autor.)

Ponga un gancho en el espacio al inicio de la opción que mejor describa su respuesta a cada pregunta. Por favor marque solo una respuesta por pregunta.

¿Cuál es su género?

Masculino _ Femenino

¿En qué rango de edad se encuentra?

\begin{tabular}{|c|c|c|}
\hline 18 a 22 años & 23 a 27 años & 28 a 32 años \\
\hline 38 a 42 años & 43 a 47 años & 48 a 52 años \\
\hline
\end{tabular}

¿Dónde reside?

_ Panamá Centro

Panamá Oeste
San Miguelito Panamá Norte

\section{Panamá Este}

Colón

1. ¿Su clase de inglés se enfoca en cómo funciona dicho idioma y los significados que se pueden transmitir a través del mismo?

_ Muy de acuerdo

_ De acuerdo Parcialmente de acuerdo

En desacuerdo Muy en desacuerdo

2. ¿En su clase se enfatiza la interacción en inglés?

Muy de acuerdo

De acuerdo

Parcialmente de acuerdo

En desacuerdo Muy en desacuerdo

3. ¿Durante las clases, se utilizan materiales e información auténtica en inglés (periódicos, panfletos, mapas, películas, revistas, canciones, noticias, etc.)?

Muy de acuerdo

De acuerdo

Parcialmente de acuerdo

En desacuerdo

Muy en desacuerdo

4. ¿Se aprende inglés haciendo y a través de la práctica directa en su clase?

Muy de acuerdo

En desacuerdo
De acuerdo Parcialmente de acuerdo

Muy en desacuerdo 
5. ¿El aprendizaje centrado en el estudiante se practica en su clase de inglés? En otras palabras, ¿`on los estudiantes mayormente los que hablan y llevan a cabo diferentes actividades en vez de tener al docente hablando o explicando algo todo el tiempo?

_ Muy de acuerdo

De acuerdo

Parcialmente de acuerdo

En desacuerdo

Muy en desacuerdo

6. ¿En su clase se hacen esfuerzos por practicar la comunicación auténtica (para el mundo real) en inglés?

Muy de acuerdo

De acuerdo

Parcialmente de acuerdo

En desacuerdo

Muy en desacuerdo

7. ¿El trabajo en parejas y el trabajo en grupos son comunes en su clase de inglés?

Muy de acuerdo

De acuerdo

Parcialmente de acuerdo

En desacuerdo

Muy en desacuerdo

8. ¿Se combina el aprendizaje del idioma con el aprendizaje de contenidos de otros temas (ciencias, negocios, deportes o historia, por ejemplo) en su clase de inglés?

Muy de acuerdo

_ De acuerdo

Parcialmente de acuerdo

En desacuerdo

Muy en desacuerdo

9. ¿Su clase de inglés se enfoca en el intercambio de información a través de la comunicación?

Muy de acuerdo

De acuerdo

Parcialmente de acuerdo

En desacuerdo

Muy en desacuerdo

10. ¿Existen resultados o metas de aprendizaje relacionados con el uso del idioma para necesidades de la vida diaria que han sido establecidos y que se espera que cumpla en su clase de inglés?

Muy de acuerdo

_ De acuerdo

Parcialmente de acuerdo

En desacuerdo

Muy en desacuerdo

11. ¿En su clase se practican y se integran las habilidades de entender, hablar, leer y escribir en inglés a través del aprendizaje cooperativo entre estudiantes?

Muy de acuerdo

De acuerdo

Parcialmente de acuerdo

En desacuerdo

Muy en desacuerdo

12. ¿El/La profesor/a es un facilitador/a de la comunicación en su clase de inglés?

Muy de acuerdo

De acuerdo

Parcialmente de acuerdo 
13. ¿El/La profesor/a fomenta el uso del inglés y es un/a participante activo/a en la clase? Muy de acuerdo De acuerdo Muy en desacuerdo
Parcialmente de acuerdo

En desacuerdo

\section{- Muy en desacuerdo}

14. ¿El/La profesor/a proporciona retroalimentación continua con respecto a los objetivos de aprendizaje de la clase?

_ Muy de acuerdo

De acuerdo

Parcialmente de acuerdo

En desacuerdo

Muy en desacuerdo

15. ¿En su clase de inglés los estudiantes son participantes comunicativos activos?

_ Muy de acuerdo

En desacuerdo
De acuerdo

Muy en desacuerdo

16. ¿Los estudiantes colaboran entre sí para el aprendizaje del idioma en su clase de inglés?

Muy de acuerdo

En desacuerdo
De acuerdo

Muy en desacuerdo
Parcialmente de acuerdo

17. ¿Además del aprendizaje en colaboración, los estudiantes de su clase de inglés practican el aprendizaje autónomo (iniciado y dirigido por ellos mismos)?

_Muy de acuerdo

_ De acuerdo

Parcialmente de acuerdo

En desacuerdo
Muy en desacuerdo

18. ¿Se practican en su clase de inglés actividades de aprendizaje colaborativo en que se debe buscar, intercambiar y compartir información?

_ Muy de acuerdo

De acuerdo

Parcialmente de acuerdo

En desacuerdo

Muy en desacuerdo

19. ¿Se dan en su clase de inglés actividades orientadas al desempeño de tareas o actividades de la vida real?

_ Muy de acuerdo

_ De acuerdo Parcialmente de acuerdo

En desacuerdo Muy en desacuerdo

20. ¿En su clase de inglés se llevan a cabo actividades dirigidas a la práctica del inglés y al desarrollo de fluidez en dicha lengua?

Muy de acuerdo Muy en desacuerdo

De acuerdo Parcialmente de acuerdo En desacuerdo 


\section{ANEXO 2: CUESTIONARIO Y PRUEBA DE VALIDEZ DE ENCUESTA}

A continuación, el cuestionario utilizado por el panel de jueces expertos para evaluar la validez de la Encuesta.

Estimado(a) Profesor(a):

Con base en los criterios presentados a continuación, se le solicita dar su opinión sobre el instrumento de recolección de datos adjunto (Encuesta \#1). Según su opinión, marque Sí o $\mathbf{N O}$ con una $\mathbf{X}$, para cada criterio.

\begin{tabular}{|c|c|c|c|}
\hline CRITERIOS & $\begin{array}{l}\text { SÍ } \\
1\end{array}$ & $\begin{array}{l}\text { NO } \\
0\end{array}$ & OBSERVACIÓN \\
\hline $\begin{array}{l}\text { 1. El instrumento recoge información que } \\
\text { permite determinar el grado de utilización de la } \\
\text { enseñanza integrada (comunicativa) de las } \\
\text { habilidades lingüísticas (escuchar, hablar, leer y } \\
\text { escribir) en un programa de inglés. }\end{array}$ & & & \\
\hline $\begin{array}{l}\text { 2. El instrumento mide lo que se supone que mida } \\
\text { solamente: el grado de utilización de la } \\
\text { enseñanza integrada (comunicativa) de las } \\
\text { habilidades lingüísticas (escuchar, hablar, leer y } \\
\text { escribir) en un programa de inglés. }\end{array}$ & & & \\
\hline 3. El formato del instrumento es adecuado. & & & \\
\hline $\begin{array}{l}\text { 4. Los ítems del instrumento cubren diversas } \\
\text { dimensiones de la variable "enseñanza integrada } \\
\text { (comunicativa) de las habilidades lingüísticas". }\end{array}$ & & & \\
\hline $\begin{array}{l}\text { 5. La secuencia en que se presentan los ítems es } \\
\text { adecuada. }\end{array}$ & & & \\
\hline $\begin{array}{l}\text { 6. Los ítems del instrumento son claros, para ser } \\
\text { entendidos y contestados por estudiantes de } \\
\text { inglés en edades de } 18-55 \text { años. }\end{array}$ & & & \\
\hline $\begin{array}{l}\text { 7. El número de ítems es adecuado para la } \\
\text { aplicación del instrumento en } 5-10 \text { minutos. }\end{array}$ & & & \\
\hline
\end{tabular}

SUGERENCIAS:

FIRMA DEL JUEZ EXPERTO(A)

NOMBRE DEL JUEZ EXPERTO(A) 
A continuación, la matriz de tabulación de acuerdos y desacuerdos del panel de jueces expertos en relación con la Encuesta.

\begin{tabular}{|c|c|c|c|c|c|c|c|c|c|c|c|c|c|c|c|c|c|c|}
\hline \multirow{3}{*}{$\begin{array}{l}\text { Criterio } \\
\mathrm{S}\end{array}$} & \multicolumn{17}{|c|}{ Jueces } & \multirow{3}{*}{$\begin{array}{l}\begin{array}{l}\text { Valo } \\
\mathrm{r} \\
\mathrm{P}\end{array} \\
\end{array}$} \\
\hline & $\mathrm{J}$ & $\mathrm{J}$ & $\mathrm{J}$ & $\mathrm{J}$ & $\mathrm{J}$ & $\mathrm{J}$ & $\mathrm{J}$ & $\mathrm{J}$ & $\mathrm{J}$ & J1 & $\mathrm{J} 1$ & J1 & J1 & $\mathrm{J} 1$ & $\mathrm{~J} 1$ & $\mathrm{~J} 1$ & $\mathrm{~J} 1$ & \\
\hline & 1 & 2 & 3 & 4 & 5 & 6 & 7 & 8 & 9 & 0 & 1 & 2 & 3 & 4 & 5 & 6 & 7 & \\
\hline 1 & 1 & 1 & 1 & 1 & 1 & 1 & 1 & 1 & 1 & 1 & 1 & 1 & 1 & 1 & 1 & 1 & 1 & 17 \\
\hline 2 & 1 & 1 & 1 & 1 & 1 & 1 & 1 & 1 & 1 & 1 & 1 & 1 & 1 & 1 & 1 & 1 & 1 & 17 \\
\hline 3 & 1 & 1 & 1 & 1 & 0 & 0 & 1 & 1 & 1 & 1 & 1 & 1 & 1 & 1 & 1 & 1 & 1 & 15 \\
\hline 4 & 1 & 1 & 1 & 1 & 1 & 1 & 1 & 1 & 1 & 1 & 1 & 1 & 1 & 1 & 1 & 1 & 1 & 17 \\
\hline 5 & 1 & 1 & 1 & 1 & 1 & 0 & 1 & 1 & 1 & 1 & 1 & 1 & 1 & 1 & 1 & 1 & 1 & 16 \\
\hline 6 & 1 & 1 & 1 & 1 & 1 & 0 & 1 & 1 & 1 & 0 & 1 & 1 & 1 & 1 & 0 & 1 & 1 & 14 \\
\hline 7 & 1 & 1 & 1 & 1 & 1 & 1 & 1 & 1 & 1 & 1 & 1 & 1 & 1 & 1 & 1 & 1 & 1 & 17 \\
\hline Total & 7 & 7 & 7 & 7 & 6 & 4 & 7 & 7 & 7 & 6 & 7 & 7 & 7 & 7 & 6 & 7 & 7 & 113 \\
\hline
\end{tabular}

Prueba de concordancia entre los jueces utilizando la fórmula

$b=(T a \div T a+T d) \times 100$

Procesamiento:

$\mathrm{Ta}=113\left(\mathrm{~N}^{\circ}\right.$ TOTAL DE ACUERDO DE JUECES)

$T d=6\left(\mathrm{~N}^{\circ}\right.$ TOTAL DE DESACUERDO DE JUECES $)$

$b=(113 \div 113+6) \times 100=94.957 \%$ de acuerdo entre los jueces 